\title{
Current misunderstandings in the management of ulcerative colitis
}

Thomas Ochsenkühn, ${ }^{1}$ Geert D'Haens ${ }^{2}$

${ }^{1}$ Department of Gastroenterology, IBD center of the University of Munich, Campus Grosshadern, Munich, Germany

${ }^{2}$ Department of Gastroenterology, Academic Medical Centre, Amsterdam, The Netherlands

\section{Correspondence to}

Geert D'Haens, Department of Gastroenterology, Academic

Medical Centre, Meibergdreef 9-C2-112, 1105 AZ Amsterdam, The Netherlands;

g.dhaens@amc.uva.nl

Published Online First 19 April 2011

\section{ABSTRACT}

Past and ongoing therapeutic concepts for ulcerative colitis have only been moderately successful.

A significant proportion of patients with ulcerative colitis will still have to undergo colectomy and overall half of the patients do not achieve sustained remission, leading to impairment of physical and mental health, social life, employment issues and sexual activity. Reluctance to treat patients early on with sufficiently potent drug regimens is obvious. Several popular misconceptions might have led to this situation. First, ulcerative colitis is still considered a more 'benign' disease than Crohn's disease. Furthermore, the general assumption is often that colectomy can 'cure' the disease. Mucosal healing as a therapeutic target has not been widely accepted. Finally, the use of antitumour necrosis factor antibodies in ulcerative colitis has been low because this treatment is considered to be less effective than in Crohn's disease. In the current review we try to disprove these misunderstandings by discussing relevant studies showing how harmful this disease can be and explaining why future studies targeting sustained suppression of inflammation could have an enormous impact on the natural course of the disease. Until these studies are available, we encourage physicians to intensify and maintain treatment until sustained remission and mucosal healing has been reached.

\section{INTRODUCTION}

Ulcerative colitis (UC) is a chronic inflammatory bowel disease of uncertain aetiology, with an incidence of $0.5-24.5$ per 100000 individuals per year ${ }^{1}$ and the highest estimates observed in the age group $20-35 .^{2}$ The site of inflammation is the mucosal layer of the colon, but occasionally deeper layers can become inflamed. The disease almost always involves the rectum and from there continuously more proximal parts of the large bowel. At diagnosis, one-third of all patients with UC have distal colitis or proctosigmoiditis, onethird have left-sided colitis and one-third more proximal or pan-colitis. ${ }^{3} 4$ Between $15 \%$ and $30 \%$ of all patients with left-sided disease suffer from extension to more proximal colonic segments. ${ }^{3} 4$ The prevailing symptoms depend on the anatomic extent and the severity of inflammation. Most patients experience recurrent episodes of rectal bleeding, passage of mucus, increased stool frequency, abdominal cramps and pain. More severe cases may develop fever, anaemia and weight loss. The combination of symptoms, endoscopic appearance and radiography determine the severity of the disease. In many patients longstanding chronic inflammation leads to structural damage of the large bowel ${ }^{5}{ }^{6}$ with impaired function and an enhanced risk of colorectal cancer. ${ }^{7}$ Aminosalicylates, corticosteroids, purine analogues and infliximab are the cornerstones of medical treatment for UC. The efficacy of adalimumab is currently under investigation. Between $5 \%$ and $50 \%$ of patients undergo colectomy during the course of the disease, mostly with an ileal pouch-anal anastomosis (IPAA). ${ }^{8}$ The wide variation in surgical rates can be explained by the availability of biological treatments, local and national guidelines and cultural differences.

The management of UC is undergoing significant changes. One aspect that has received more attention lately is the importance of mucosal healing. Already in 1923, however, Sir Arthur F Hurst, one of the first authors to describe UC, ${ }^{9}$ concluded in an article $^{10}$ that "no case of UC can be regarded as cured until the sigmoidoscope shows that the mucous membrane is perfectly healthy'. This treatment goal got lost or seems to have been ignored in the meantime by several generations of physicians. A century later, the role of mucosal integrity has again moved into the centre of interest.

Another paradigm shift has brought more attention to maintenance treatment. While it was generally accepted for decades that patients with UC only need treatment when symptoms occur, the idea of continued treatment that minimises or eliminates inflammation and reduces the risk of relapse now finds increasing support. ${ }^{11}$ The idea that the destructive effect of chronic inflammation in the gut is ongoing, even if patients do not have major symptoms is, however, well founded. ${ }^{12}$

National guidelines that have been developed in Europe and elsewhere have mainly focused on when and how to use steroids, aminosalicylates and surgery. ${ }^{13}$ Only late in the course of the disease, immunosuppressants and tumour necrosis factor (TNF) blockers have been introduced rather hesitantly, even long after their advent on the market. The main concerns relate to the potential toxicity of the newer agents without paying attention to the harm caused by prolonged corticosteroid use or undertreatment. In a number of European countries there is the assumption among gastroenterologists and surgeons that TNF blockers should be offered only as an alternative to colectomy.

As the medical professionals taking care of patients with UC, we have to admit that our 
treatment concepts have not been very successful. Ongoing disease activity is present in $\sim 50 \%$ of all patients with UC, colectomy rates remain high, and absence from social activities, unemployment, impaired quality of life, sick leave and disability pensions are higher in patients with UC than in the general population. ${ }^{14}$

Could it be that misunderstandings and misconceptions have led to this rather unsuccessful outcome? The current paper is an attempt to list and critically review our potential misunderstandings and strategic views and to offer guidelines for more optimal care in the future.

\section{CURRENT MISUNDERSTANDINGS UC is a 'benign' disease: colectomy, mortality and remission rates}

The assumption that UC is a 'benign' disease can easily be refuted by the current colectomy rates. European studies recently reported a 10-year cumulative colectomy risk of $9 \%{ }^{15}$ with a slight gradient from northern to southern European centres. $^{15}{ }^{16}$ In a paediatric cohort study in 113 children aged $0-17$ years, the calculated cumulative rate of colectomy was even $20 \%$ at 5 years. ${ }^{17}$ It takes a lot of euphemism to call a disease 'benign' when it leads to organ loss in 1 in 10 patients.

In addition, the so far largest meta-analysis ${ }^{18}$ of $>10000$ patients with UC in 10 studies recently found a greater mortality risk in patients with more than distal UC as compared with the respective background population (RR 1.2, 95\% CI 1.0 to 1.5 , $\mathrm{p}=0.047)$. The majority of patients with UC present with more than distal colitis, as was shown in the IBSEN cohort. ${ }^{3}$ Of 519 patients, 33\% had proctitis, $35 \%$ left-sided colitis and $32 \%$ extensive colitis at diagnosis. In another large meta-analysis, ${ }^{19}$ patients with UC were also found to be at an increased risk of cancer of the liver and biliary system (RR 2.58, 95\% CI 1.58 to 4.22), an observation possibly related to the relatively frequent occurrence of primary sclerosing cholangitis in patients with UC.

In the landmark study by Langholz et al, that looked at the outcomes of patients with UC over 25 years from 1962 until 1987 (when aminosalicylates and steroids were the only drugs used), only $25 \%$ of patients in the $3-7$ years after first diagnosis were in remission, $57 \%$ of patients had intermittent and $18 \%$ had continuous activity. ${ }^{20}$ Within a median observation period of 3 years, $77 \%$ of the patients had at least one second flare. Colectomy rates at 10 years were $24 \%$.

As already mentioned, in the Norwegian IBSEN cohort study 423 patients with UC were asked to categorise their clinical course for the previous 10 years using one out of four predefined curves (figure 1). Twelve per cent of patients could not choose a curve because they had already been colectomised. The remaining patients selected curve 3 or 4 in $43 \%$ of the cases, which means that $\sim 50 \%$ of the patients had an unfavourable course of the disease (either colectomy or chronic continuous or chronic intermittent symptoms). It is difficult to understand why the authors concluded that patients with newly diagnosed UC have an overall good prognosis during the first 10 years of disease.

In an interesting recent survey in North America, 450 patients with UC and 300 gastroenterologists were asked independently how they perceived the impact of UC. ${ }^{21}$ Patients reported, on average, eight flares per year, while gastroenterologists estimated the annual relapse frequency at three. Sixty-two per cent of patients with UC reported that their disease made it difficult to lead a normal life, compared with gastroenterologists' estimations of $36 \%$. Most surprisingly, only $42 \%$ of patients believed that being in remission could mean living without symptoms. The same authors compared the answers to health-related questions of the patients with UC with those of $>900$ patients with rheumatoid arthritis, asthma and migraine. ${ }^{22}$ Patients with UC reported significantly more worries about disease complications, depression and embarrassment than patients with the other chronic conditions.

Another way to look at severity of UC is to look at therapeutic outcome following treatment for an acute relapse. In many clinical trials, investigators have used 'response' rates as an end point, whereas recent guidelines have recommended using more strict criteria such as remission rates in combination with mucosal healing. Physicians advising patients on their therapeutic options based on results in clinical trials must be aware that most indices used in these trials did not define remission as the total absence of any symptoms. ${ }^{23}$ For example in the ACT trials, ${ }^{24}$ remission was defined as a Mayo score of up to 2 points with no individual subscore exceeding 1 point, which means that patients could be considered in remission in the presence of mild disease activity with subtle bleeding or diarrhoea.

Three typical patient populations have been examined in clinical trials for UC: (1) the mostly untreated patients with a mild to moderate flare; (2) mesalamine-refractory patients; and (3) steroidand/or immunosuppressant-refractory patients.

In patients with a flare of mild to moderate UC who had not been treated with $>2$ g of mesalazine the two most recent global UC trials with a new mesalazine preparation were performed by Kamm and Lichtenstein and later published again in a combined analysis. ${ }^{25-27}$ Of the 517 patients, 8 -week complete mucosal healing rates were $35 \%$ and $37 \%$ with two doses of mesalazine (4.8 and $2.4 \mathrm{~g} /$ day), compared with $18 \%$ on placebo. This means that although $40 \%$ had mild disease and $80 \%$ had only left-sided colitis, only about one-third of these patients was brought into remission after 8 weeks of treatment. If a sigmoidoscopy end point score of 0 had been chosen-that is, a healthy mucosa-less than half of these patients would have reached remission. This means that the ideal outcome of a symptom-free state with fully healed mucosa is achieved in $<20 \%$ of cases in this type of patient. 

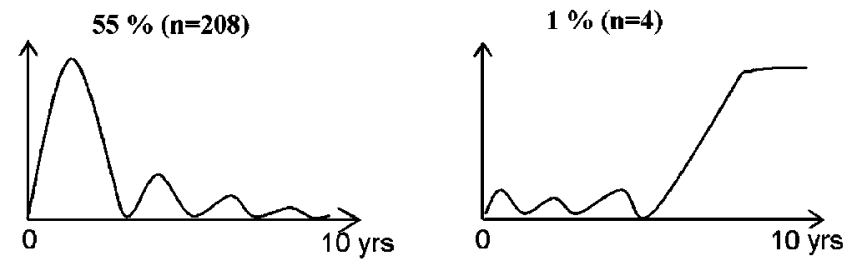

Curve1: Remission or mild severity of intestinal symptoms after initial high activity

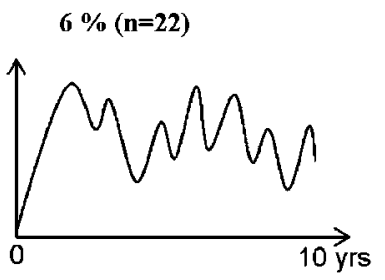

Curve 3: Chronic continuous symptoms
Curve 2: Increasc in the scverity of intestinal symptoms after initial low activity

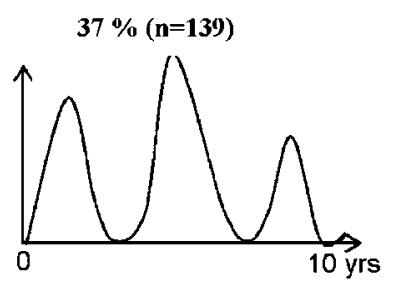

Curve 4: Chronic intermittent symptoms

Figure 1 Results of the Norwegian IBSEN cohort study. A total of 423 patients with ulcerative colitis were asked to categorise their clinical course for the previous 10 years using one out of four predefined curves (reproduced from Solberg et al ${ }^{3}$ ).

If mesalazine fails, systemic steroids are usually introduced. In such a situation, remission rates at 30 days were $54 \%$, based on a retrospective analysis describing the natural history of steroid treatment for UC. ${ }^{28}$ Mucosal healing after treatment with corticosteroids has not been investigated. ${ }^{29}$

More than 700 patients with active UC who were either steroid dependent or refractory, or refractory to immunosuppressives were treated with the TNF-blocking agent infliximab (Remicade, Centocor/MSD) in the ACT trials. ${ }^{24}$ Remission rates at week 30 were as low as $30 \%$ and $36 \%$ for 5 and $10 \mathrm{mg} / \mathrm{kg}$ infliximab, respectively. Surprisingly, mucosal healing rates at the same time point were as high as $48 \%$ and $53 \%$, with mucosal healing defined as a Mayo subscore for endoscopy of 0 or 1 . Patients with mucosal healing (Mayo grade 0) at week 8 were more likely to be in clinical remission at week $30 .{ }^{30}$ In both ACT trials, the most potent anti-inflammatory agent currently available, infliximab, brought only one out of three patients into remission, not taking into consideration that one-third of these patients were still receiving steroids in addition.

In summary, the perception that UC is a benign disease is not justified. UC is a disease that leads to organ loss in $5-25 \%$ of cases, is associated with increased mortality and has a negative impact on daily life. The drugs that we use and the way in which we use them do not bring the majority of affected patients into 'remission', which is the most desirable outcome. UC is a potentially aggressive, mostly undertreated and sometimes lethal chronic disease. Only a minority of patients experience a disease course that can be called 'benign'.

\section{UC can be 'cured' by colectomy}

When all medical treatment options have been exhausted, patients with intractable or badly

controllable UC usually undergo colectomy. A total proctocolectomy with IPAA $^{8}$ has become the procedure of choice for intractable UC and also in the case of dysplasia or cancer. Some authors have even suggested to 'move up' surgery to an earlier therapeutic step as a form of 'top-down' approach. ${ }^{31}$ Although it is often stated that by removing the colon normal life can be restored in all patients, this is unfortunately rarely the case.

Studies that assessed health-related quality of life in a postcolectomy UC population generally reported an improvement after surgery. However, most of these surveys looked at patients with the most severe forms of UC and are characterised by a rather short-term follow-up. ${ }^{32} 33$

Looking a bit further down the line, however, a variety of complications can occur after surgery. Pouchitis, pouch leakage, pelvic abscesses, pouch fistulae, small bowel obstruction, anastomotic stricture, postoperative bleeding, faecal incontinence, sexual dysfunction and female infertility are frequent. A recent retrospective analysis of the Leuven IBD group in $>180$ patients with UC who underwent proctocolectomy in 1990-2004 described the outcome after IPAA. ${ }^{34}$ Within 1 month after closure of ileostomy, $27 \%$ of all patients developed at least one complication. After a median follow-up time of 6.5 years, sepsis was seen in $14 \%$ and obstructive complications in $26 \%$ of all patients, respectively, with $5 \%$ of the patients having both septic and obstructive complications. Another retrospective chart review of 31 paediatric patients with UC who underwent colectomy reported obstructive complications in $16 \%$ and fistulas in $16 \%$ within a mean observation period of 4.5 years. $^{35}$

\section{The most common complication is pouchitis}

In the Leuven cohort $^{34}$ (median follow-up of 6.5 years), $46 \%$ of patients developed at least one episode of acute pouchitis. Of these patients, $40 \%$ developed fewer than three episodes of pouchitis during follow-up, $19 \%$ had acute relapsing pouchitis and $41 \%$ developed chronic pouchitis. This means that relapsing or chronic inflammation in the pouch occurred in $>1$ in 4 of all patients with IPAA. Their global pouch failure rate was $5 \%$. In a German single centre cohort review, pouch failure rates of even $13 \%$ at a median follow-up of 12 years were reported. ${ }^{36}$ Lichtenstein and colleagues stated that 1 in 6 of their patients with IPAA required reversal and had a conventional ileostomy. ${ }^{37}$

Furthermore, even with a 'successful' pouch, functional results are far from the 'normal situation'. In the Leuven cohort, ${ }^{34} 50 \%$ of patients with IPAA had more than five bowel movements per day and $25 \%$ had more than two bowel movements at night. Soiling or seepage at night was reported by $15 \%$ and the IBD-related ongoing need for continuous or occasional medication by $35 \%$. In a recently published Swiss study with a similar design, 107 patients were evaluated for their functional results after IPAA within a median follow-up period of 7 years. ${ }^{38}$ Sixty-six per cent of patients reported 
5-10 bowel movements per day and 73\% had at least one movement during the night. Twenty-five per cent had episodes of soiling. Night time continence was excellent in only $41 \%$ of patients.

In a recent meta-analysis ${ }^{39}$ of seven studies, female fertility in 481 patients with IPAA was compared with that in 411 patients with UC without IPAA. In the surgical group, the risk of infertility was increased from $15 \%$ to $48 \%$. The authors concluded that these findings may encourage physicians to consider even potentially hazardous rescue treatments including ciclosporin and infliximab, before submitting young women to colectomy. Indeed, if at all possible, pouch construction should be postponed until a female patient has had as many pregnancies as desired.

In summary, proctocolectomy with ileoanal pouch for UC is often not the final solution. At present, it remains the best possible solution for patients with 'intractable' UC, preferably after exhaustion of all medical options. Patients should be informed, however, about what to expect after this surgery in terms of quality of life, potential complications and need for further drug treatment. Winslow indeed reported that issues such as diet, sexual function and returning to work are often neglected in the discussion with patients who need to make choices regarding IPAA surgery. ${ }^{40}$ Quality of life has also been reported to improve with infliximab treatment, but no direct comparisons with surgery have been performed. ${ }^{41}$

\section{Anti-TNF antibodies are less effective in UC than in Crohn's disease (CD)}

The reluctance to use anti-TNF antibodies in UC is often based on the (subjective) assumption that infliximab works better in CD than in UC. However, the three major controlled trials which examined the effect of infliximab in luminal (ACCENT I) and fistulising (ACCENT II) CD and in UC (ACT) showed a different picture. ${ }^{29} 4243$

Clinical remission rates at 1 year in the three studies were $35 \%$ in the ACT trial, $33 \%$ in the ACCENT-I trial and $36 \%$ in the ACCENT-II trial. Taking into account that patients in the ACT trial started on the study with more immunosuppressives $(50 \%)$ and corticosteroids $(60 \%)$ than patients in the ACCENT I (33\% and 50\%) and ACCENT II trials $(33 \%$ and $30 \%$ ), it appears that infliximab is equally effective in UC and CD. In addition, antibody formation against infliximab or low trough levels might also play a role in the efficacy of infliximab. In UC and CD there is a lack of data investigating the influence of trough levels or antibody formation; however, in rheumatoid arthritis, the clinical response to infliximab closely follows the trough drug levels and the presence of antibodies directed against the drug. ${ }^{44}$

Mucosal healing rates were also comparable in all trials. In another trial with patients refractory to intravenous steroids or whose only alternative was colectomy, infliximab led to complete clinical and endoscopic remission in $40 \%(6 / 15)$ of all patients. $^{45}$
The efficacy of another anti-TNF antibody, adalimumab (Humira, Abbott), in UC was less convincing. ${ }^{46}$ In a trial with a design similar to the ACT trials, 8 week remission rates were only $19 \%$ in the study group that received adalimumab every other week versus $9 \%$ in the placebo group. Longterm results for this trial are being awaited. Novel biologicals such as golimumab (another anti-TNF antibody) and vedolizumab (a humanised monoclonal antibody against the $\alpha 4-\beta 7$-integrin) are currently being tested in UC.

In conclusion, at least for infliximab the efficacy of biological treatment has been comparable with that in CD.

\section{Mucosal healing is not so important in UC}

Mucosal healing has recently become a pivotal therapeutic goal in CD as well as in UC. Mucosal healing had been defined by the International Organization of IBD (IOIBD) ${ }^{47}$ as the absence of friability, blood, erosions and ulcers in all visualised segments of the colonic mucosa. The majority of clinical trials used this definition, although disappearance of the normal vascular pattern is usually considered compatible with mucosal healing.

Prospective studies in $\mathrm{CD}^{48}$ and retrospective studies in $\mathrm{UC}^{49}$ have already confirmed the benefit of early and sustained mucosal healing for the patients' global health. In the recent Norwegian study by Frøslie et $a l^{49}$ the authors examined the impact of mucosal healing on the subsequent course of disease in $>350$ patients with UC, before biological treatment was available. Mucosal healing after 1 year of treatment was significantly associated with a low risk of colectomy of $2 \%$ at 5 years. Patients without mucosal healing were colectomised 3.5 times more frequently. Most interestingly, this association was independent from the clinical disease course within the 5 years of observation (remission after initial activity, continuous activity, relapsing disease or accelerating disease activity). The follow-up study ${ }^{50}$ of Järnerot's infliximab trial $^{45}$ achieved a similar result: none of eight patients in endoscopic remission at 3 months had a colectomy within a follow-up period of 3 years, compared with $50 \%$ of patients who were not in remission $(\mathrm{p}=0.02)$.

Achievement of mucosal healing has been associated with better outcomes, less surgery and less hospitalisation. An analysis of the Leuven cohort of patients with UC treated with infliximab showed that colectomy was more frequent if patients did not achieve mucosal healing at week 4 or $10 .{ }^{51}$ In one subanalysis of the ACT trials, it was shown that patients treated with infliximab were less likely to undergo colectomy during 54 weeks than those receiving placebo. ${ }^{52}$ As mentioned above, in another subanalysis of the ACT data, patients with mucosal healing at week 8 were in remission at week 30 in $48 \%$ as compared with those without mucosal healing who only reached remission in $10 \%{ }^{30}$

Consequently, mucosal healing appears to represent a highly relevant therapeutic end point in 
patients with UC. ${ }^{11}$ Evaluation of mucosal inflammation in UC is easy, since the disease is mostly present in the rectum, and flexible sigmoidoscopy or proctoscopy is possible even without extensive bowel cleansing. Furthermore, faecal calprotectin levels correlate well with endoscopically evaluated disease activity and reliably discriminate inactive from mild disease, moderate disease and highly active disease. ${ }^{53}$

In summary, past and ongoing treatment concepts for ulcerative colitis have only been moderately successful. Five to $25 \%$ of patients still lose their colon and overall only half of the patients achieve sustained remission. Reluctance to treat patients early on with sufficiently potent drug regimens and with mucosal healing as a therapeutic goal may offer a partial explanation for this phenomenon. Future studies on therapeutic strategies should aim at sustained suppression of inflammation, which perhaps could lead to a profound change in the course of the disease. In the meantime physicians should be encouraged to intensify and maintain treatment until sustained mucosal healing is reached.

\section{Competing interests None.}

Provenance and peer review Commissioned; externally peer reviewed

\section{REFERENCES}

1. Lukas M, Bortlik M, Maratka Z. What is the origin of ulcerative colitis? Still more questions than answers. Postgrad Med J 2006;82:620-5.

2. Loftus EV Jr. Clinical epidemiology of inflammatory bowel disease: incidence, prevalence, and environmental influences. Gastroenterology 2004;126:1504-17.

3. Solberg IC, Lygren I, Jahnsen J, et al. Clinical course during the first 10 years of ulcerative colitis: results from a population-based inception cohort (IBSEN Study). Scand J Gastroenterol 2009:44:431-40.

4. Hoie 0, Wolters FL, Riis L, et al. Low colectomy rates in ulcerative colitis in an unselected European cohort followed for 10 years. Gastroenterology 2007:132:507-15.

5. Moum B, Ekbom A, Vatn MH, et al. Change in the extent of colonoscopic and histological involvement in ulcerative colitis over time. Am J Gastroenterol 1999:94:1564-9.

6. Niv $\mathbf{Y}$, Bat $L$, Ron $E$, et al. Change in the extent of colonic involvement in ulcerative colitis: a colonoscopic study. $\mathrm{Am} \mathrm{J}$ Gastroenterol 1987;82:1046-51.

7. Rutter M, Saunders B, Wilkinson K, et al. Severity of inflammation is a risk factor for colorectal neoplasia in ulcerative colitis. Gastroenterology 2004;126:451-9.

8. Fazio VW, Ziv Y, Church JM, et al. Ileal pouch-anal anastomoses complications and function in 1005 patients. Ann Surg 1995;222:120-7.

9. Hurst AF. Ulcerative colitis. Guy's Hospital Rep 1909;71:26

10. Hurst AF. Discussion on ulcerative colitis. Proc $R$ Soc Med 1923;16:106-8.

11. Pineton de Chambrun G, Peyrin-Biroulet $L$, Lémann $M$, et al. Clinical implications of mucosal healing for the management of IBD. Nat Rev Gastroenterol Hepatol 2010;7:15-29.

12. Osada T, Ohkusa T, Okayasu I, et al. Correlations among total colonoscopic findings, clinical symptoms, and laboratory markers in ulcerative colitis. J Gastroenterol Hepatol 2008;(23 Suppl 2): S262-7.

13. Hoffmann JC, Zeitz M, Bischoff SC, et al. Diagnosis and therapy of ulcerative colitis: results of an evidence based consensus conference by the German Society of Digestive and Metabolic Diseases and the competence network on inflammatory bowel disease. Z Gastroenterol 2004:9:979-1007.

14. Bernklev T, Jahnsen J, Henriksen M, et al. Relationship between sick leave, unemployment, disability, and health-related quality of life in patients with inflammatory bowel disease. Inflamm Bowel Dis 2006;12:402-12

15. Henriksen $\mathbf{M}$, Jahnsen J, Lygren I, et al. Ulcerative colitis and clinical course: results of a 5 -year population-based follow-up study (the IBSEN study). Inflamm Bowel Dis 2006; 12:543-50.

16. Jürgens $\mathbf{M}$, Laubender RP, Hartl F, et al. Disease activity, ANCA and IL23R genotype status determine early response to infliximab in patients with ulcerative colitis. Am J Gastroenterol 2010;105:1811-19

17. Gower-Rousseau C, Dauchet L, Vernier-Massouille G, et al. The natural history of pediatric ulcerative colitis: a population-based cohort study. Am J Gastroenterol 2009;104:2080-8.

18. Jess T, Gamborg M, Munkholm P, et al. Overall and causespecific mortality in ulcerative colitis: meta-analysis of population-based inception cohort studies. Am J Gastroenterol 2007:102:609-17.

19. Pedersen N, Duricova D, Elkjaer M, et al. Risk of extra-intestinal cancer in inflammatory bowel disease: meta-analysis of population-based cohort studies. Am J Gastroenterol 2010;105:1480-7.

20. Langholz E, Munkholm P, Davidsen M, et al. Course of ulcerative colitis: analysis of changes in disease activity over years. Gastroenterology 1994;107:3-11.

21. Rubin DT, Siegel CA, Kane SV, et al. Impact of ulcerative colitis from patients' and physicians' perspectives: results from the UC: NORMAL survey. Inflamm Bowel Dis 2009;15:581-8.

22. Rubin DT, Dubinsky MC, Panaccione $R$, et al. The impact of ulcerative colitis on patients' lives compared to other chronic diseases: a patient survey. Dig Dis Sci 2010;55:1044-52.

23. D'Haens G, Sandborn WJ, Feagan BG, et al. A review of activity indices and efficacy end points for clinical trials of medical therapy in adults with ulcerative colitis. Gastroenterology 2007:132:763-86.

24. Rutgeerts P, Sandborn WJ, Feagan BG, et al. Infliximab for induction and maintenance therapy for ulcerative colitis. N Engl J Med 2005;353:2462-76.

25. Kamm MA, Sandborn WJ, Gassull M, et al. Once-daily, high concentration MMX mesalamine in active ulcerative colitis. Gastroenterology 2007;132:66-75.

26. Lichtenstein GR, Kamm MA, Boddu $P$, et al. Effect of once- or twice-daily MMX mesalamine (SPD476) for the induction of remission of mild-to-moderately active ulcerative colitis. Clin Gastroenterol Hepatol 2007;5:95-102.

27. Sandborn WJ, Kamm MA, Lichtenstein GR, et al. MMX Multi Matrix System mesalazine for the induction of remission in patients with mild-to-moderate ulcerative colitis: a combined analysis of two randomized, double-blind, placebo-controlled trials. Aliment Pharmacol Ther 2007;26:205-15.

28. Faubion WA Jr, Loftus EV Jr, Harmsen WS, et al. The natural history of corticosteroid therapy for inflammatory bowel disease: a population-based study. Gastroenterology 2001;121:255-60.

29. Van Assche G, Vermeire S, Rutgeerts P. Mucosal healing and anti TNFs in IBD. Curr Drug Targets 2010;11:227-33.

30. Rutgeerts P, Sandborn W, Reinisch W, et al. Gastroenterology. 2005;128(4) (abstract).

31. Siegel CA. Making therapeutic decisions in inflammatory bowel disease: the role of patients. Curr Opin Gastroenterol 2009;25:334-8.

32. Weinryb RM, Gustavsson JP, Liljeqvist $L$, et al. A prospective study of the quality of life after pelvic pouch operation. J Am Coll Surg 1995;180:589-95.

33. McLeod RS, Churchill DN, Lock AM, et al. Quality of life of patients with ulcerative colitis preoperatively and postoperatively. Gastroenterology 1991;101:1307-13.

34. Ferrante M, Declerck S, De Hertogh G, et al. Outcome after proctocolectomy with ileal pouch-anal anastomosis for ulcerative colitis. Inflamm Bowel Dis 2008;14:20-8.

35. Patton D, Gupta N, Wojcicki JM, et al. Postoperative outcome of colectomy for pediatric patients with ulcerative colitis. J Pediatr Gastroenterol Nutr 2010:51:151-4.

36. Leowardi C, Hinz U, Tariverdian M, et al. Long-term outcome 10 years or more after restorative proctocolectomy and ileal pouch-anal anastomosis in patients with ulcerative colitis. Langenbecks Arch Surg 2010;395:49-56.

37. Lichtenstein GR, Cohen R, Yamashita B, et al. Quality of life after proctocolectomy with ileoanal anastomosis for patients with ulcerative colitis. J Clin Gastroenterol 2006;40:669-77.

38. Wuthrich P, Gervaz P, Ambrosetti P, et al. Functional outcome and quality of life after restorative proctocolectomy and ileo-anal pouch anastomosis. Swiss Med Wkly 2009;139:193-7. 
39. Waljee A, Waljee J, Morris AM, et al. Threefold increased risk of infertility: a meta-analysis of infertility after ileal pouch-anal anastomosis in ulcerative colitis. Gut 2006;55:1575-80.

40. Winslow F. Follow-up of patients with ileo-anal pouch following stoma reversal. Gastroint Nurs 2006;4:16-23.

41. Feagan BG, Reinisch W, Rutgeerts $P$, et al. The effects of infliximab therapy on health-related quality of life in ulcerative colitis patients. Am J Gastroenterol 2007:102:794-802.

42. Hanauer SB, Feagan BG, Lichtenstein GR, et al. Maintenance infliximab for Crohn's disease: the ACCENT I randomised trial. Lancet 2002;359:1541-9.

43. Sands BE, Anderson FH, Bernstein CN, et al. Infliximab maintenance therapy for fistulizing Crohn's disease. N Engl J Med 2004:350:876-85

44. Radstake TR, Svenson M, Eijsbouts AM, et al. Formation of antibodies against infliximab and adalimumab strongly correlates with functional drug levels and clinical responses in rheumatoid arthritis. Ann Rheum Dis 2009;68:1739-45.

45. Järnerot G, Hertervig E, Friis-Liby I, et al. Infliximab as rescue therapy in severe to moderately severe ulcerative colitis: a randomized, placebo-controlled study. Gastroenterology 2005:128:1805-11.

46. Reinisch W, Sandborn W, Hommes D, et al. Adalimumab for induction of clinical remission in moderately to severely active ulcerative colitis: results of a randomised controlled trial. Gut 2011;60:780-7.

47. Allez M, Lémann M. Role of endoscopy in predicting the disease course in inflammatory bowel disease. World $\mathrm{J}$ Gastroenterol 2010;16:2626-32.

48. Baert F, Moortgat L, Van Assche G, et al. Mucosal healing predicts sustained clinical remission in patients with early-stage Crohn's disease. Gastroenterology 2010;138:463-8.

49. Froslie KF, Jahnsen J, Moum BA, et al. Mucosal healing in inflammatory bowel disease: results from a Norwegian population-based cohort. Gastroenterology 2007;133:412-22.

50. Gustavsson A, Järnerot $G$, Hertervig $E$, et al. Clinical trial: colectomy after rescue therapy in ulcerative colitis-3-year follow-up of the Swedish-Danish controlled infliximab study. Aliment Pharmacol Ther 2010;32:984-9.

51. Ferrante $\mathbf{M}$, Vermeire $\mathrm{S}$, Fidder $\mathrm{H}$, et al. Long-term outcome after infliximab for refractory ulcerative colitis. J Crohns Colitis 2008:2:219-25.

52. Sandborn WJ, Rutgeerts $P$, Feagan BG, et al. Colectomy rate comparison after treatment of ulcerative colitis with placebo or infliximab. Gastroenterology 2009;137:1250-60.

53. Schoepfer AM, Beglinger C, Straumann A, et al. Ulcerative colitis: correlation of the Rachmilewitz endoscopic activity index with fecal calprotectin, clinical activity, C-reactive protein, and blood leukocytes. Inflamm Bowel Dis 2009:15:1851-8.

Advancing Postgraduates. Enhancing Healthcare.

The Postgraduate Medical Journal is dedicated to advancing the understanding of postgraduate medical education and training.

- Acquire the necessary skills to deliver the highest possible standards of patient care

- Develop suitable training programmes for your trainees

- Maintain high standards after training ends

Published on behalf of the fellowship for Postgraduate Medicine

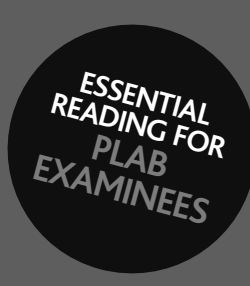

FOR MORE DETAILS OR TO SUBSCRIBE, VISIT THE WEBSITE TODAY

\section{postgradmedj.com}




\section{GUT Current misunderstandings in the management of ulcerative colitis}

Thomas Ochsenkühn and Geert D'Haens

Gut 2011 60: 1294-1299 originally published online April 19, 2011

doi: $10.1136 /$ gut.2010.218180

Updated information and services can be found at:

http://gut.bmj.com/content/60/9/1294

\section{These include:}

References This article cites 51 articles, 5 of which you can access for free at: http://gut.bmj.com/content/60/9/1294\#BIBL

\section{Email alerting} service

Receive free email alerts when new articles cite this article. Sign up in the box at the top right corner of the online article.

Topic Articles on similar topics can be found in the following collections Collections

\section{Notes}

To request permissions go to:

http://group.bmj.com/group/rights-licensing/permissions

To order reprints go to:

http://journals.bmj.com/cgi/reprintform

To subscribe to BMJ go to:

http://group.bmj.com/subscribe/ 